\title{
Blade Tip-timing Technology with Multiple Reference Phases for Online Monitoring of High-speed Blades under Variable-speed Operation
}

\author{
Zhang Ji-wang ${ }^{1}$, Zhang Lai-bin ${ }^{2}$, Ding Ke-Qin ${ }^{1}$, Duan Li-xiang ${ }^{2}$ \\ ${ }^{1}$ China Special Equipment Inspection and Research Institute, Beijing, 100029, China, 121889248@qq.com \\ ${ }^{2}$ College of Mechanical and Transportation Engineering, China University of Petroleum, Beijing 102249, China
}

\begin{abstract}
High-speed blades form core mechanical components in turbomachines. Research concerning online monitoring of operating states of such blades has drawn increased attention in recent years. To this end, various methods have been devised, of which, the blade tip-timing (BTT) technique is considered the most promising. However, the traditional BTT method is only suitable for constant-speed operations. But in practice, the rotational speed of turbomachine blades is constantly changing under the influence of external factors, which lead to unacceptable errors in measurement. To tackle this problem, a new BTT method based on multi-phases is proposed. A plurality of phases was arranged as evenly as possible on the rotating shaft to determine the rotation speed. Meanwhile, the corresponding virtual reference point was determined in accordance with the number of blades between consecutive phases. Based on these reference points, equations to measure displacement due to blade vibrations were deduced. Finally, mathematical modeling, numerical simulation and experimental tests were performed to verify the validity of the proposed method. Results demonstrate that the error in measurement induced when using the proposed method is less than $1.8 \%$, which is much lower compared to traditional methods utilized under variable-speed operation.
\end{abstract}

Keywords: BTT technology, variable speed, online monitoring, multiple reference phases (MRP).

\section{INTRODUCTION}

Rotating blades constitute core mechanical components in turbomachine systems. These blades operate under the influence of high speeds and strong centrifugal forces, causing severe blade vibrations and may even lead to disastrous consequences. Therefore, there exists a need for continuous monitoring of the blade state under various operating conditions. Development of online-monitoring methods for rotating blades has become an important area of research in recent years, and various techniques, such as the strain-gauge technology [1], optical backscatter reflectometer [2], laser Doppler [3], acoustic emission [4], coherent laser radar [5], and blade-tip timing [6]-[8] have been devised. Amongst these methods, the BTT technology is considered to be the most promising owing to its advantages of low cost and non-contact utility [9]-[11]. The BTT technique is based on measuring the difference between the theoretical and actual times recorded by blade-sweeping probes. The vibration amplitude, phase, and frequency information may be subsequently obtained using specific algorithms [12]. Most extant studies concerning BTT systems have either focused on BTT sensor technology [13]-[16] or blade vibration parameter identification [17]-[21] while still considering blade operation at a constant rotating speed. In practice, however, the rotational speed in turbomachines is hardly constant owing to continuous variations in working conditions and external effects, thereby rendering traditional BTT systems no longer usable in practical turbomachine applications.

Presently, a lot of research is being performed on the development of monitoring and diagnosis systems for use under variable-speed operation of turbomachines, and these studies, at present, mainly focus on bearings and gearboxes [22]-[27]. It has been reported in [28], [29] that continuous monitoring of high-speed blades is difficult to achieve under variable-speed operation; however, no further research has been performed in this regard. Work reported in [30] introduced a measurement method based on the one phase method (OP); however, its induced error in measurement was unacceptably large owing to large fluctuations in rotating speed.

In view of the above problems, the authors, in this paper, propose a new BTT method based on multiple reference phases, wherein a plurality of reference phases is arranged on the rotating shaft, thereby providing estimates of the rotational speed. Several virtual phases are then inserted to ensure each blade has a reference phase. Subsequently, theoretical derivation of blade vibrations is performed based 
on the above method, and the vibration-displacement equation corresponding to variable-speed operation is obtained. Consequently, a new mathematical model for determining blade vibration is established to verify the feasibility of the method.

\section{CONVENTIONAL BLADE TIP-TIMING SYSTEM}

The BTT technique is a non-contact blade vibration measuring method, the underlying measurement principle of which is depicted in Fig.1. Several probes (probes 1, 2, and 3 depicted in the figure) are embedded into a stationary casing around a disk with $\mathrm{K}$ blades (here, 35 blades are used as example.). The arrival time of each blade tip crossing these probes is recorded by means of a series of pulse signals. An additional probe sensor $\mathrm{S}$ is installed as a reference probe close to the shaft. When no blade vibrations are induced, the expected blade-tip arrival time intervals must be constant. Once vibrations are set up, a difference is induced between the theoretical and actual times. This difference in times is recorded by the probes for further analysis.

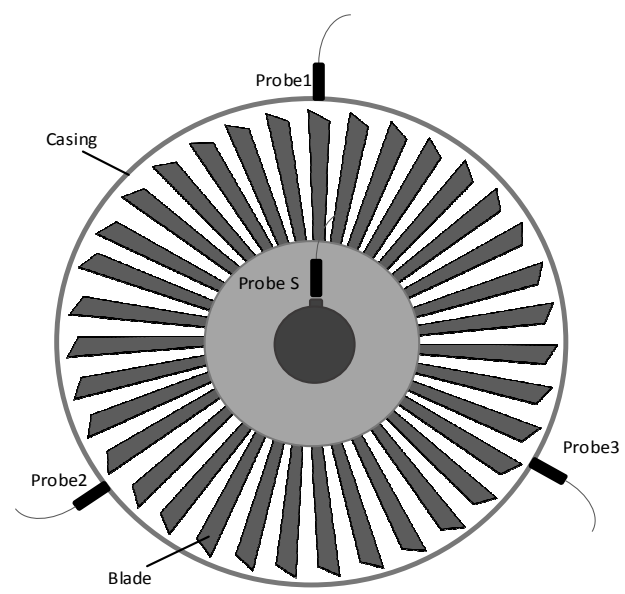

Fig.1. Measurement principle of traditional BTT systems.

Displacements corresponding to blade vibrations could be calculated using (1).

$$
\mathrm{y}_{\text {in }}=\left(t_{a}-t_{b}\right) * V
$$

Here, $t_{a}, t_{b}$ represent the actual and the theoretical bladetip arrival times, respectively, and $V$ is the linear velocity of blade tip.

The basic principle underlying the BTT technique is to measure the difference between theoretical and actual times at which the blade tips sweep across the probes, thereby facilitating the calculation of vibration displacement. This implies that irrespective of whether the measuring method depends on the reference phase or not [8], the rotational speed must be maintained constant. However, in practical turbomachine applications, the speed of rotation hardly remains constant. This limits the usability of traditional BTT systems in practical turbomachine applications. For the sake of convenience, the non-phase method is hereinafter referred to as NP in this manuscript.

\section{BLADE VIBRATION MEASUREMENT USING MULTIPLE REFERENCE PHASES}

In order to tackle the problem of blade monitoring under variable-speed operation, a multiple reference phase (MRP) method is proposed, and the vibration displacement formula based on virtual phase interpolation has been derived.

\subsection{Principle}

The principle of the MRP method is depicted in Fig.2. Reference phases are arranged as evenly as possible on the rotating shaft. It must be noted that the number of reference phases is far lower compared to the number of blades owing to structural limitations. If the number of blades is an integral multiple of the number of reference phases, the reference phases need to be evenly distributed, as depicted in Fig.2.a). Even distribution of reference phases is also required in order to achieve an accurate measurement. The difference in blade number between adjacent phases must not exceed 1, as shown in Fig.2.b). The speed between two adjacent phases of rotation could be considered constant, since change in speed is a continuous process. In the same manner, several virtual phases could be inserted based on the number of blades, thereby ensuring each blade has a reference point.

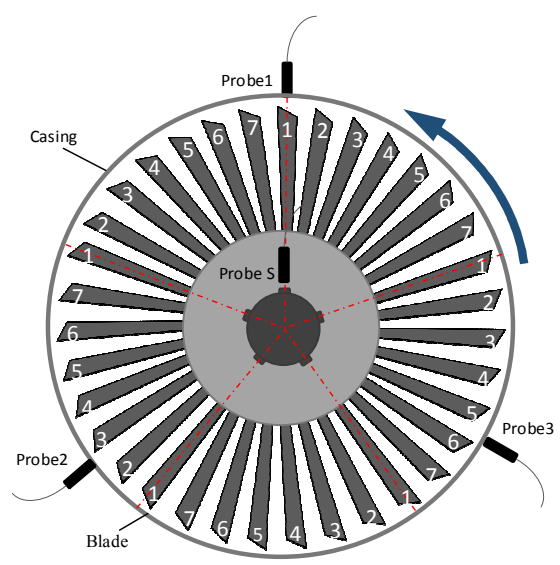

a) Uniform distribution of reference phases

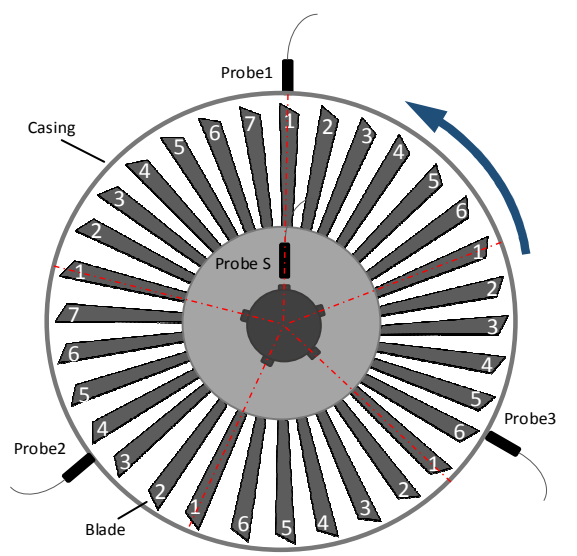

b) Non-uniform distribution of reference phases

Fig.2. Measurement principle of BTT system with multiple reference phases. 
Regardless of the uniformity of reference-phase distribution, the proposed method is capable of performing accurate measurements (section 4). For the sake of convenience, the uniform phase distribution is considered when deriving the measurement equation. The equation, however, is equally applicable for non-uniform distributions as well. Assuming a turbine system with $N$ blades and $n$ reference phases, $m$ blades are considered to exist between adjacent phases. The corresponding principle of measurement is depicted in Fig.3. A total of $m-1$ virtual reference phases are inserted between adjacent phase signals at equal intervals. The vibration displacement corresponding to each blade could be calculated using the difference between the actual blade-sweeping time recorded by the probes and the corresponding virtual reference phase time.

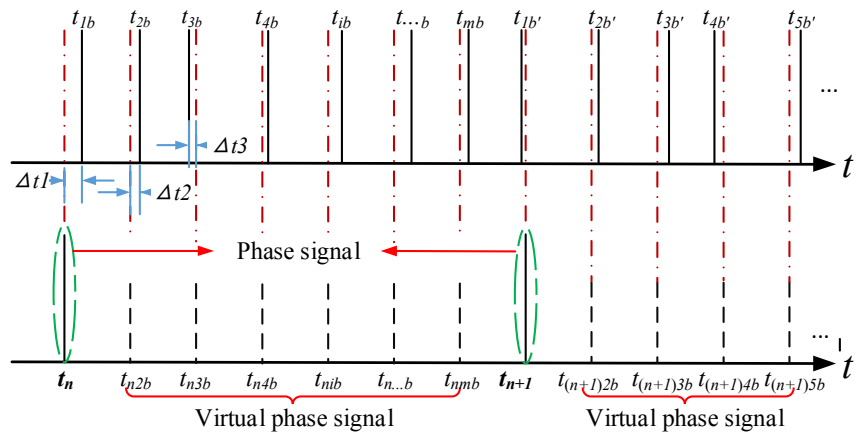

Fig.3. Schematic of BTT measuring method based on multiple reference phases.

Fig.3. is divided into upper and lower parts. The upper part is the blade tip timing sequence, and the lower part is the reference phase timing sequence. Because the number of reference phases is far lower than the number of blades, virtual phase interpolation is needed between the reference phases according to the number of blades contained, so that each blade has a corresponding reference phase. Here, $t_{n}$ and $t_{n+1}$ represent actual phase-measuring times while $t_{n i b}$ represents the virtual phase time; $t_{i b}$ is the actual measuring time of the $i$-th blade; $\Delta t_{i}$ represents the difference between the actual measuring time and the theoretical arrival time of the $i$-th blade.

\subsection{Vibration displacement of blade tips}

As shown in Fig.2. and Fig.3., the blade vibration displacement can be expressed in the form of equation (2). Considering the possible inherent errors, the value $y_{d}$ is introduced, which can be obtained via static calibrations.

$$
\mathrm{y}_{\text {in }}=\Delta t_{i} * V_{t}-y_{d}
$$

Here, $y_{\text {in }}$ represents the vibration amplitude of the $i$-th blade, $\Delta t_{i}$ represents the difference between the actual arrival and theoretical times of the $i$-th blade, and $V_{t}$ expresses linear velocity of the blade tip.
It is assumed that the system comprises $n$ reference phases with $m$ blades between adjacent phases. The basic principle underlying the measurement of blade-vibration displacement is depicted in Fig.3., employing which (3), (4), and (5) may be deduced.

$$
\begin{gathered}
\Delta t_{i}=t_{i b}-t_{n i b} \\
V_{t}=\frac{2 \pi R}{T}=\frac{1}{n} * \frac{2 \pi R}{t_{n+1}-t_{n}} \\
y_{i n}=\left(t_{i b}-t_{n i b}\right) * \frac{1}{n} * \frac{2 \pi R}{t_{n+1}-t_{n}}-y_{d}
\end{gathered}
$$

In the above equations, $T$ represents the period of rotation while $t_{n}$ and $t_{n+1}$ represent actual arrival times of successive phases. With respect to Fig.3., the virtual phase time can be described by (6).

$$
t_{n i b}=t_{n}+\frac{i-1}{m} *\left(t_{n+1}-t_{n}\right) \quad(i \leq m)
$$

Equation (5) could be expressed as

$y_{i n}=\left\{t_{i b}-\left[t_{n}+\frac{i-1}{m} *\left(t_{n+1}-t_{n}\right)\right]\right\} * \frac{1}{n} * \frac{2 \pi R}{t_{n+1}-t_{n}}-y_{d}$

Also, equation (7) may be simplified to the following form.

$$
y_{i n}=\left(t_{i b}-t_{n}\right) * \frac{1}{n} * \frac{2 \pi R}{t_{n+1}-t_{n}}-\frac{i-1}{m} * \frac{1}{n} * 2 \pi R-y_{d}
$$

Here $\frac{i-1}{m} * \frac{1}{n} * 2 \pi R-y_{d}$ has a constant value, represented by $y_{d}^{\prime}$; thus, equation (9) takes the following form

$$
y_{i n}=\frac{2 \pi R\left(t_{i b}-t_{n}\right)}{n\left(t_{n+1}-t_{n}\right)}-y_{d}^{\prime}
$$

Thus, the equation to evaluate blade-vibration displacement may be derived based on the proposed MRP method, thereby facilitating quick and accurate calculation of vibration displacements.

\section{VALIDATION}

\subsection{Mathematical modeling}

The low-order vibration equation for high-speed rotating blades [7], [8] is given by

$$
y=A \cos (\omega t+\varphi)
$$

The time-domain data concerning blade vibrations were converted to corresponding values in the angular domain in order to deduce a more accurate expression. With regard to blade vibrations in the angle domain, the measured angle in radians represents the sum of the rotation and blade-vibration angles. Equation (11) may, therefore, be written as 


$$
\theta=\theta_{r}+\theta_{v^{\prime}}
$$

Where $\theta_{r}$ represents the cumulative angular movement of the rotating shaft and can be described as

$$
\theta_{r}=\omega t=\sum_{i=0}^{i=t} \omega_{r i}
$$

$\theta_{v}$, on the other hand, represents the blade-vibration angle and may be written as

$$
\theta_{v^{\prime}}=\frac{A \cos (\omega t+\varphi)}{R}=\frac{A \cos \left(2 \pi f_{v} t+\varphi\right)}{R}
$$

Therefore, equation (11) becomes

$$
\theta=\theta_{r}+\theta_{v^{\prime}}=\sum_{i=0}^{i=t}\left(\omega_{r i}+\frac{A \cos \left(2 \pi f_{v} t+\varphi\right)}{R}\right)
$$

Considering the proposed system requirement of minimum sampling resolution, the variable $t$ has been discretized as demonstrated in (15).

$$
t=\frac{N}{10^{9}}
$$

The angle $\theta$ may, therefore, be expressed as

$$
\theta=\sum_{i=0}^{i=t} \omega_{r i}+\sum_{n=0}^{n=N} \frac{A \cos \left(2 \pi f_{v} * \frac{n}{10^{9}}+\varphi\right)}{R}
$$

Three forms of speed fluctuations in rotating machinesuniform variable speed, periodic fluctuation, and random fluctuation, have been considered in the analysis presented herein.

\section{1) Uniform variable speed process}

The uniform variable speed process can be expressed using the following expression.

$$
\begin{gathered}
\omega_{r i}=\omega_{0}+a t \\
\sum_{i=0}^{i=t} \omega_{r i}=\sum_{n=0}^{n=N}\left(\omega_{0}+a * \frac{n}{10^{9}}\right)
\end{gathered}
$$

Using the above equations, $\theta$ may be evaluated as

$$
\theta=\sum_{n=0}^{n=N}\left[\omega_{0}+a * \frac{n}{10^{9}}+\frac{A \cos \left(2 \pi f_{v} * \frac{n}{10^{9}}+\varphi\right)}{R}\right]
$$

2) Periodic fluctuation

Periodic fluctuations in speed may be represented as

$$
\begin{gathered}
\omega_{r i}=\omega_{0} * \sin \left(\omega_{1} t+\varphi_{1}\right) \\
\sum_{i=0}^{i=t} \omega_{r i}=\sum_{n=0}^{n=N} \omega_{0} * \sin \left(2 \pi f_{1} * \frac{n}{10^{9}}+\varphi_{1}\right)
\end{gathered}
$$

In this case, $\theta$ takes the following form.

$\theta=\sum_{n=0}^{n=N}\left[\omega_{0} \sin \left(2 \pi f_{1} * \frac{n}{10^{9}}+\varphi\right)+\frac{A \cos \left(2 \pi f_{v} * \frac{n}{10^{9}}+\varphi\right)}{R}\right]$

3) Random fluctuation

Lastly, random speed fluctuations may be expressed using the following equation.

$$
\omega_{i}=\omega_{0} \text { uifrnd }\left(n_{1}, n_{2}\right)
$$

Equation (16), therefore, takes the following form.

$\theta=\omega_{0}$ unifrnd $\left(n_{1}, n_{2}\right)+\sum_{n=0}^{n=N}\left[\frac{A \cos \left(2 \pi f_{v} * \frac{n}{10^{9}}+\varphi\right)}{R}\right]$

By assuming a uniform distribution of $z$ reference phases, the sampling time is considered as the first point with meeting the following equation (23).

$$
\theta_{r} \geq k\left(\frac{2 \pi}{z}\right) \quad(k=1,2,3, \ldots)
$$

The vibration frequency of the blade was assumed to be $134 \mathrm{~Hz}$; correspondingly, the amplitude of vibration was set as $0.01 \mathrm{~mm}$. To make calculations convenient, all initial phases were set to zero. Five reference phases were uniformly distributed. Following points must be noted.

1) For the uniform acceleration process, the rotational speed was found to increase from 0 to $6000 \mathrm{rpm}$ in 5 seconds.

2) For periodic speed fluctuations, the fluctuation frequency $f_{1}$ was set as $25 \mathrm{~Hz}$.

3) For random speed fluctuations, the range of fluctuations was set from 0.95-1.05 times the fundamental frequency.

Subsequently, blade-vibration displacements under different operating conditions were obtained and are depicted in Fig.4.

As depicted in Fig.4., regardless of the type of speedfluctuation model used, measurement errors induced in the non-phase (NP) and one-phase (OP) methods cannot be ignored. The NP method demonstrated the largest error in measurement (of the order of $200 \%$ and above). 
Corresponding errors in the OP method were found to increase with increase in the number of blade passages and were typically of the order of $50 \%$. On the other hand, the proposed MRP method demonstrated high measuring accuracy, and the maximum error in measurement was found to be of the order of $1.8 \%$. This confirms the ability of the proposed method to greatly improve the accuracy and range of applicability of the BTT system.

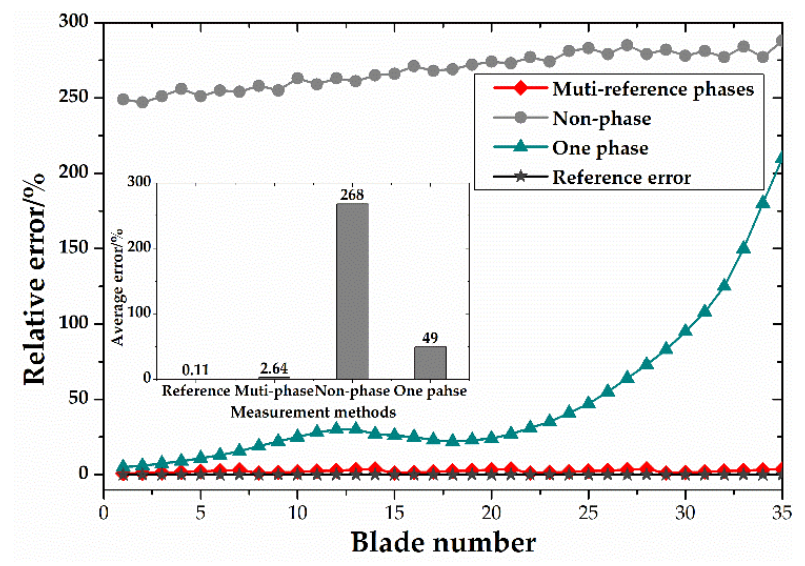

a) Case with uniformly variable speed

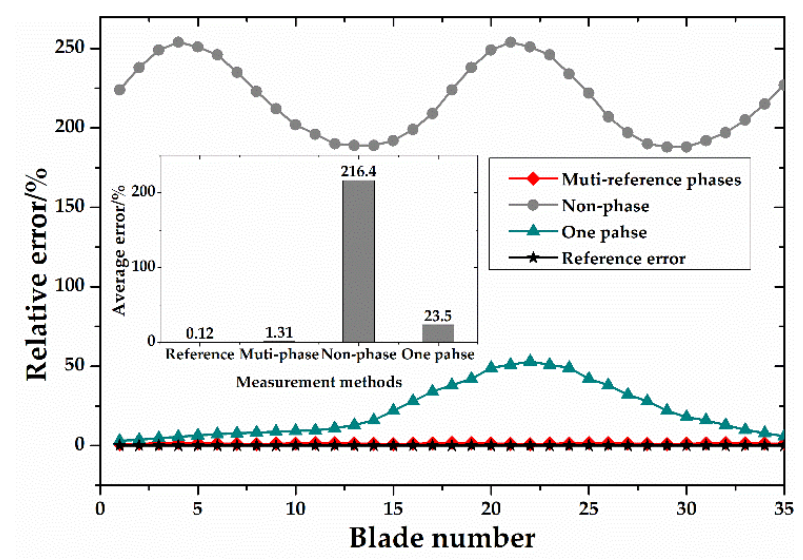

b) Case with periodic speed fluctuations

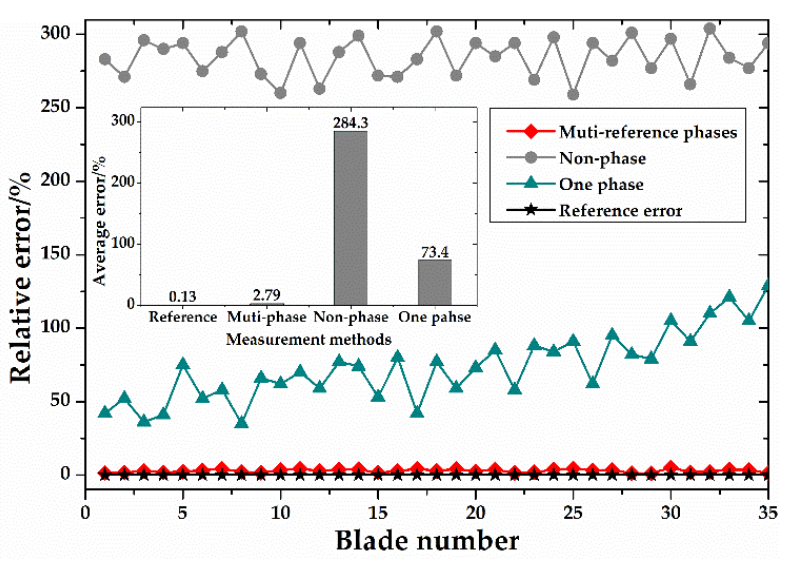

c) Case with random speed fluctuation
Fig.4. Comparison of errors in measurement induced under different speed-fluctuation models.

\subsection{Numerical simulation}

Numerical simulations, using the ADAMS software, were also performed under variable-speed operating conditions (starting-up process) to verify the validity of the proposed method. The ADAMS software offers great advantages in dynamic analysis, and has been recognized by both, industry as well as academia [31]. The simulation model was designed as depicted in Fig.5. Three timing sensors were installed evenly on the casing to record blade arrival times. An additional sensor was fixed close to the rotating shaft with five reference phases to record reference arrival times. Blade vibration response signals were obtained by simulation as depicted in Fig.6. The whole starting-up process lasted 3 seconds, and the speed of rotation was increased from 0 to $6000 \mathrm{rpm}$. Sampling data were subsequently obtained based on the three uniform monitoring methods (BTT sampling principle described in section 3.1). Other parameters are listed in Table 1.

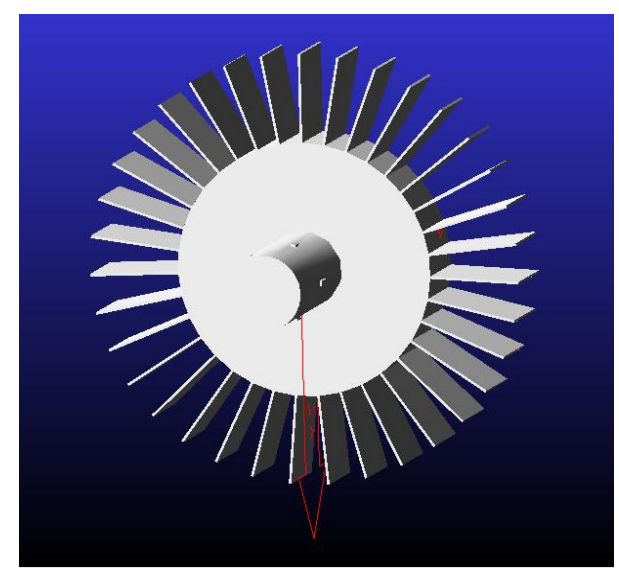

Fig.5. Simulation model.

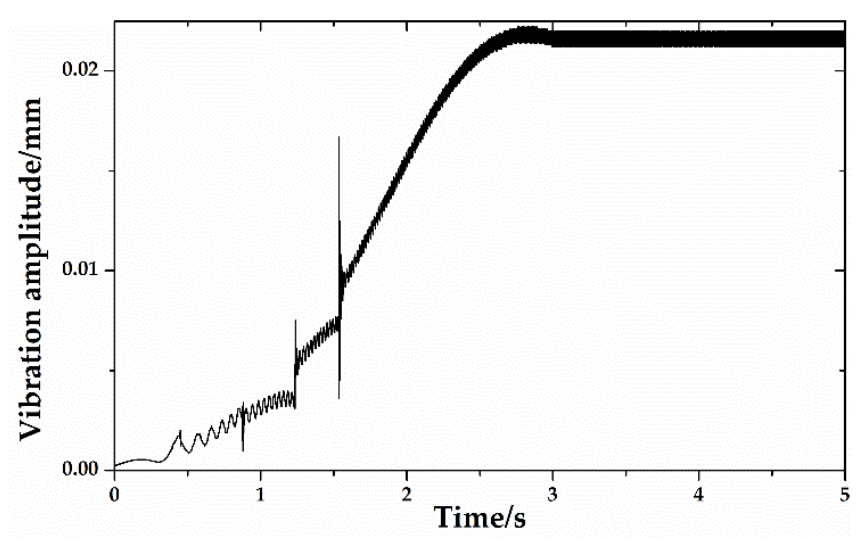

Fig.6. Simulation result.

Vibration response data concerning all blades were collected and corresponding vibration displacements were measured using the NP, OP, and MRP methods. Subsequently, the relative errors in measurement induced by 
the various measurement methods are obtained and plotted as depicted in Fig. 7.

Table 1. Numerical simulation parameters.

\begin{tabular}{|l|l|}
\hline Parameters & Properties \\
\hline Material & Type 45 steel \\
\hline The number of blades & 35 \\
\hline The number of reference phases & 5 \\
\hline The length of blade & $110 \mathrm{~mm}$ \\
\hline Rotation radius & $230 \mathrm{~mm}$ \\
\hline The width of each blade & $60 \mathrm{~mm}$ \\
\hline Blade thickness & $2.5 \mathrm{~mm}$ \\
\hline The rotating speed & $0-6000 \mathrm{rpm}$ \\
\hline
\end{tabular}

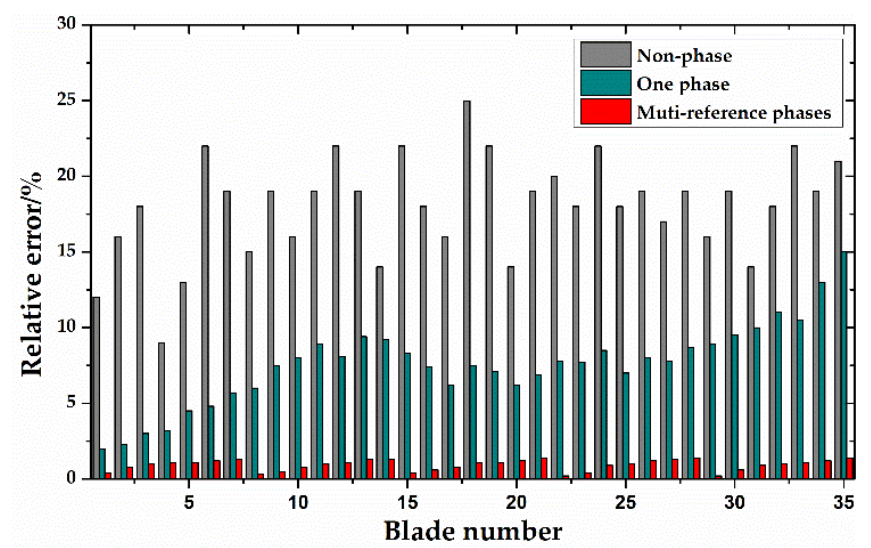

Fig.7. Comparison of relative measurement errors induced by use of different measurement methods (simulation).

As can be observed in Fig.7., the mean errors induced by the three methods-NP, OP, and MRP-during the start-up process were of the order of $18 \%, 7.8 \%$, and $1.1 \%$, respectively, implying that the error in measurement induced when using the MRP method is considerably smaller compared to that induced by traditional methods. Meanwhile, the distribution of errors was also found to be different. Errors induced by the NP method demonstrated random fluctuations, those corresponding to the OP method demonstrated increase with increasing blade number, and those induced by the MRP method demonstrated periodic fluctuations. As depicted in Fig.3., during the continuous acceleration process, $\Delta t$ was found to increase with increase in the number of blades, thereby causing a gradual increase in the measurement error. Because the circle is evenly divided into $\mathrm{N}$ parts when using the MRP method, errors in the measurement of $\Delta t$ and $V$ in (2) are reduced $\mathrm{N}$ times. Thus, the measurement accuracy has been greatly improved.

\subsection{Experimental test}

An experimental test is also built to validate the feasibility of the proposed method, shown in Fig. 8. The test-bed mainly includes electric machinery, coupling, gearbox, blades, and sensor. Four reference phases are uniformly embedded in the rotating shaft to record rotation speed, and the detailed experimental parameters are shown in Table 2.

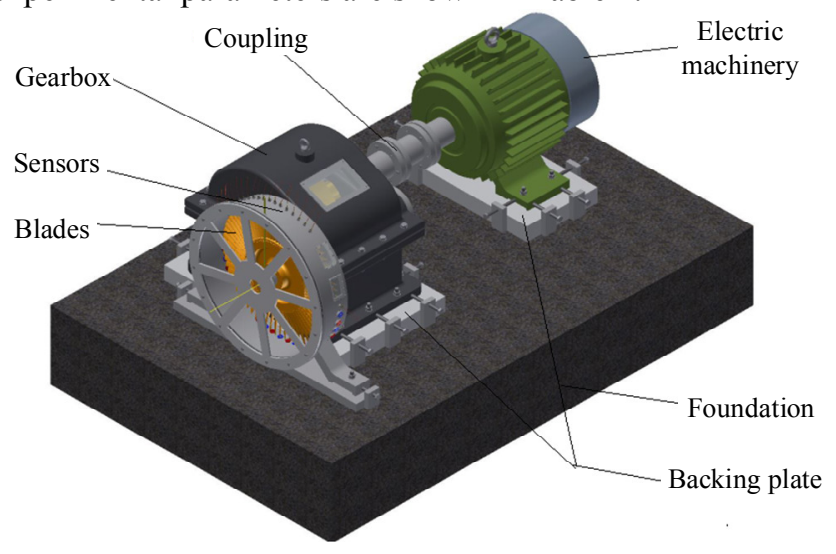

Fig.8. Test-bed.

Table 2. Experimental parameters.

\begin{tabular}{|l|l|}
\hline Parameters & Properties \\
\hline Material & Aluminum alloy \\
\hline The number of blades & 32 \\
\hline The number of reference phases & 4 \\
\hline Rotation diameter & $137 \mathrm{~mm}$ \\
\hline The rotating speed & $6000 \mathrm{rpm}$ \\
\hline Fluctuation of speed & $10 \mathrm{~Hz}$ \\
\hline
\end{tabular}

In the test, the rotation speed of blades is changed by using the speed control system. The vibration values of all blades at variable speed are also collected by using the NP, OP, and MRP methods. Finally, the relative errors of all blades by using these three measurement methods are obtained as shown in Fig.8.

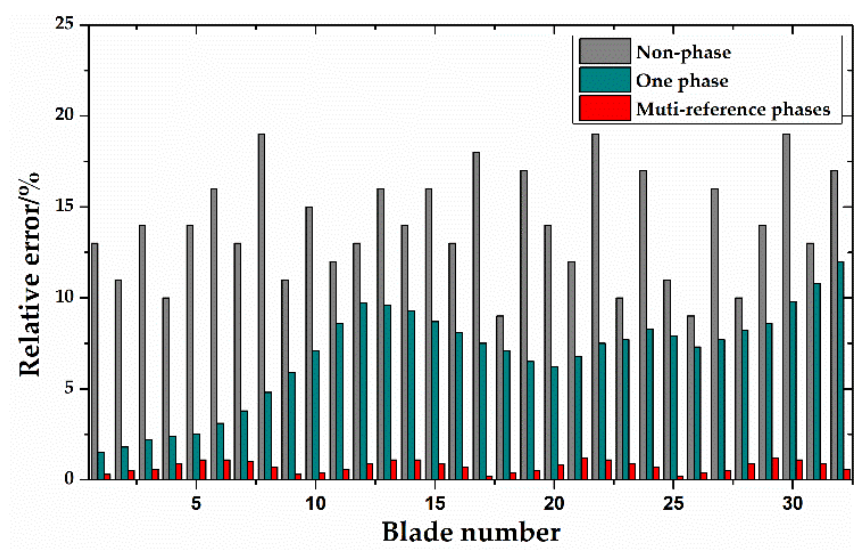

Fig.8. Comparison of relative measurement errors induced by use of different measurement methods (test).

As shown in Fig.8., the experimental results are similar to the simulation. The mean measurement errors of the NP, OP, and MRP methods are $13.9 \%, 6.8 \%$, and $0.8 \%$, 
respectively. It is obvious that the measurement error of the MRP method is much smaller than traditional methods.

Obviously, no matter the simulation or the experimental tests, the MRP method has good measurement results under variable speed operation. Because the speed change process is discretized, it greatly improves the accuracy of speed and tip timing. Thus, high-precision measurement of data can be performed using the proposed method.

\section{CONCLUSION}

The BTT method is considered as one of the most promising techniques in high-speed blade-state monitoring. However, the traditional BTT method is not applicable to variablespeed operation of typical turbomachines. To tackle this problem, a new BTT technique incorporating multiple reference phases for online monitoring of turbomachine blades operating under varying rotational speeds is proposed herein, and the corresponding measurement equations for evaluating vibration-induced blade displacements are also deduced. Finally, mathematical modeling, numerical simulations and experimental tests were performed to verify the validity of the proposed method. Results of the proposed study demonstrate that the measurement error induced when using the proposed method is less than $1.8 \%$, which is considerably lower compared to traditional methods. The major findings of and conclusions drawn from this study may be summarized as follows.

1) A new BTT technique with multiple reference phases for online monitoring of high-speed blades under variable-speed operation is proposed, and the measurement equation for the method is derived based on virtual phase interpolation.

2) The vibration equation of the rotating blade is derived by transforming the time domain to the angle domain. And the blade vibration under three different speed conditions has been deduced and discussed.

3) The feasibility and effectiveness of the proposed method and corresponding derived equations are verified via mathematical modeling, numerical simulations and experimental tests. Results demonstrate that regardless of the type of speed fluctuations involved, the measurement error induced when using the proposed method is considerably smaller compared to that induced when employing traditional methods.

\section{ACKNOWLEDGMENT}

This study was supported by the National Key R\&D Program of China (2016YFF0203302-02), National Natural Science Foundation of China (No.51674277) and the National Key Research and Development Program of China (No. 2017YFC0805803)

\section{REFERENCES}

[1] Tavakolpour-Saleh, A.R., Setoodeh, A.R., Gholamzadeh, M. (2016). A novel multi-component strain-gauge external balance for wind tunnel tests: Simulation and experiment. Sensors and Actuators A: Physical, 247, 172-186.

[2] Sierra-Pérez, J., Torres-Arredondo, M.A., Güemes, A. (2016). Damage and nonlinearities detection in wind turbine blades based on strain field pattern recognition.
FBGs, OBR and strain gauges comparison. Composite Structures, 135, 156-166.

[3] Rothberg, S.J., Allen, M.S., Castellini, P. (2017). An international review of laser Doppler vibrometry: Making light work of vibration measurement. Optics and Lasers in Engineering, 99, 11-22.

[4] Tang, J., Soua, S., Mares, C. (2016). An experimental study of acoustic emission methodology for in service condition monitoring of wind turbine blades. Renewable Energy, 99, 170-179.

[5] Talbot, J., Wang, Q., Brady, N. (2016). Offshore wind turbine blades measurement using Coherent Laser Radar. Measurement, 79, 53-65.

[6] Battiato, G., Firrone, C.M., Berruti, T.M. (2017). Forced response of rotating bladed disks: Blade TipTiming measurements. Mechanical Systems and Signal Processing, 85, 912-926.

[7] Lin, J., Hu, Z., Chen, Z.-S. (2016). Sparse reconstruction of blade tip-timing signals for multimode blade vibration monitoring. Mechanical Systems and Signal Processing, 81, 250-258.

[8] Guo, H., Duan, F., Zhang, J. (2016). Blade resonance parameter identification based on tip-timing method without the once-per revolution sensor. Mechanical Systems and Signal Processing, 66-67, 625-639.

[9] dos Santos, F.L.M., Peeters, B., van der Auweraer, H. (2016). Vibration-based damage detection for a composite helicopter main rotor blade. Case Studies in Mechanical Systems and Signal Processing, 3, 22-27.

[10] Rzadkowski, R., Rokicki, E., Piechowski, L. (2016). Analysis of middle bearing failure in rotor jet engine using tip-timing and tip-clearance techniques. Mechanical Systems and Signal Processing, 76-77, 213-227.

[11] Rigosi, G., Battiato, G., Berruti, T.M. (2017). Synchronous vibration parameters identification by tip timing measurements. Mechanics Research Communications, 79, 7-14.

[12] Neumann, M., Dreier, F., Günther, P. (2015). A laseroptical sensor system for blade vibration detection of high-speed compressors. Mechanical Systems and Signal Processing, 64-65, 337-346.

[13] Allport, J.M., Jupp, M.L., Pezouvanis, A. (2012). Turbocharger blade vibration: Measurement and validation through laser tip-timing. In 10th International Conference on Turbochargers and Turbocharging, 173-181.

[14] Chen, Z., Yang, Y., Xie, Y. (2013). Non-contact crack detection of high-speed blades based on principal component analysis and Euclidian angles using opticalfiber sensors. Sensors and Actuators A: Physical, 201, 66-72.

[15] Di Maio, D., Ewins, D.J. (2012). Experimental measurements of out-of-plane vibrations of a simple blisk design using Blade Tip Timing and Scanning LDV measurement methods. Mechanical Systems and Signal Processing, 28, 517-527.

[16] Günther, P., Dreier, F., Pfister, T. (2011). Measurement of radial expansion and tumbling motion of a high- 
speed rotor using an optical sensor system. Mechanical Systems and Signal Processing, 25, 319-330.

[17] Zhou, Z., Chen, S., Li, W. (2018). Experiment study of aerodynamic performance for the suction-side and pressure-side winglet-cavity tips in a turbine blade cascade. Experimental Thermal and Fluid Science, 90, 220-230.

[18] Ma, H., Lu, Y., Wu, Z., Tai, X. (2016). Vibration response analysis of a rotational shaft-disk-blade system with blade-tip rubbing. International Journal of Mechanical Sciences, 107, 110-125.

[19] Tahani, M., Maeda, T., Babayan, N. (2017). Investigating the effect of geometrical parameters of an optimized wind turbine blade in turbulent flow. Energy Conversion and Management, 153, 71-82.

[20] Heath, S., Imregun, M. (1996). An improved singleparameter tip-timing method for turbomachinery blade vibration measurements using optical laser probes. International Journal of Mechanical Sciences, 38, 1047-1058.

[21] Xie, F., Ma, H., Cui, C. (2017). Vibration response comparison of twisted shrouded blades using different impact models. Journal of Sound and Vibration, 397, 171-191.

[22] Huang, H., Baddour, N., Liang, M. (2018). Bearing fault diagnosis under unknown time-varying rotational speed conditions via multiple time-frequency curve extraction. Journal of Sound and Vibration, 414, 43-60.

[23] Feng, Z., Chen, X., Wang, T. (2017). Time-varying demodulation analysis for rolling bearing fault diagnosis under variable speed conditions. Journal of Sound and Vibration, 400, 71-85.

[24] Mishra, C., Samantaray, A.K., Chakraborty, G. (2016). Rolling element bearing defect diagnosis under variable speed operation through angle synchronous averaging of wavelet de-noised estimate. Mechanical Systems and Signal Processing, 72-73, 206-222.

[25] Wang, T., Liang, M., Li, J. (2015). Bearing fault diagnosis under unknown variable speed via gear noise cancellation and rotational order sideband identification. Mechanical Systems and Signal Processing, 62-63, 30-53.

[26] Abboud, D., Antoni, J., Sieg-Zieba, S. (2017). Envelope analysis of rotating machine vibrations in variable speed conditions: A comprehensive treatment. Mechanical Systems and Signal Processing, 84, 200226.

[27] Guo, Y., Li, G., Chen, H. (2017). Development of a virtual variable-speed compressor power sensor for variable refrigerant flow air conditioning system. International Journal of Refrigeration, 74, 73-85.

[28] Han, D., Pastrikakis, V., Barakos, G.N. (2016). Helicopter performance improvement by variable rotor speed and variable blade twist. Aerospace Science and Technology, 54, 164-173.

[29] Yang, J., Song, D., Dong, M. (2016). Comparative studies on control systems for a two-blade variablespeed wind turbine with a speed exclusion zone. Energy, 109, 294-309.

[30] Wang, W., Ren, S., Chen, L., Shao, H. (2017). The blade vibration measurement research based on the key phase interpolation method. Journal of Vibration, Measurement \& Diagnosis, 37, 361-365.

[31] Blundell, M., Harty, D. (2014). Multibody systems simulation software. In The Multibody Systems Approach to Vehicle Dynamics (Second Edition). Butterworth-Heinemann, 87-184

Received April 09, 2018 Accepted November 07, 2018 